the hypothesis of positive and hegative valences may possibly become a necessary adjunct to the structure theory. This, as I have stated before, ${ }^{1}$ must depend upon the extent of its applications and experimental verifications, and upon the part that is played by just criticisms in bringing to light the relative merits and demerits of its applications.

From these and the preceding points of view noted in this paper, the general criticisms and conclusions of Brunel have little, if any, bearing upon the electronic conception of positive and negative valences as a formulative hypothesis.

Cincinatit, OHIo.

[From the Harriman RESEARch Laboratory, RoOsevelt Hospital, NEW York, and the Chemical Department, Goucher College, Baltimore, Md.]

\title{
NEPHELOMETRIC ESTIMATION OF PHOSPHORUS.
}

Bx PAILIP ADOLPH KOBER AND GRETE EGERER.

Received May 28, 1915.

TABLE of Contents.-I. Introduction. II. Reagent. III. Discussion. IV. Applications. V. Summary.

\section{Introduction.}

The estimation of phosphorus in biological and industrial sciences, especially of minute amounts, is becoming of increasing importance A large number of volumetric and colorimetric methods have been proposed but thus far none has been satisfactory for micro-quantitative work. While looking for a suitable nephelometric precipitant ${ }^{2}$ for phosphorus in connection with our nuclease technic ${ }^{3}$ our attention was called ${ }^{4}$ to the reagent developed by Pouget and Chouchak. ${ }^{5}$ This reagent consists of a nitric acid solution of strychnine and molybdic acid and produces a very sensitive reaction with phosphates (one milligram of phosphorus in 2 liters giving a very marked suspension of insoluble substance) similar in sensitiveness to the Nessler or the Graves ${ }^{\theta}$ reaction for ammonia. The authors found it will detect I part of phosphorus in 20 million parts

1 This Journal, 37, 892 (1915).

${ }^{2}$ Uranium acetate and magnesia mixture were tried as nephelometric precipitants, but as preliminary experiments, especially with the latter, were not successful, these reagents were abandoned.

${ }^{3}$ P. A. Kober and S. S. Graves, This Journal, 36, 1304 (1914).

${ }^{4}$ Dr. I. Greenwald of this laboratory, after making an attempt to apply this reagent directly for nephelometric work, abandoned it. Subsequently (see J. Biol. Chem., 21, 29 ( 1915$)$ ). he recommended the original reagent for a colorimeter method, although, according to him, the color of the opalescent liquid is so slight that it is not apparent until it is put into the instrument. It is obvious that any defect in the suspension for nephelometric work will make a defect for colorimetric, and our experiments, as indicated elsewhere, show that the reagent as recommended by the original authors, although usable, is not satisfactory.

- Bull. soc. chim., 5, 104 (r909); 9, 649 (1911).

- This JouRNal, 37, I181 (1915). 
of water. As the precipitate is slightly yellow and remains in suspension for a long time, the authors recommended it as a colorimetric reagent, but, practically, their estimations were turbidimetric.

On studying the reaction carefully, we found that (I) it was not constant and quantitative ${ }^{1}$ and $(2)$ the reagent gradually become yellow and deteriorated, probably because of the action of the nitric acid. Pouget and Chouchak realized this, as their directions state that the mixing of constituents must be made only just before using. After making many variations of all constituents, no marked improvement was obtained, but on substituting hydrochloric for nitric acid, the solution not only remained practically colorless for an indefinite length of time, bit wes stable and gave quantitative and constant results.

\section{Reagent.}

(a) Variations of Constituents.-The insoluble substance formed in this reaction is probably a phosphomolybdic acid complex of strychnine. the important factor is the hydrochloric acid content of the reagent. If the amount of acid is too small, an insoluble strychnine-molybdic acid compound precipitates and if too large, the phosphate precipitate is redissolved. The latitude between these extremes is, however, quite satisfactory. Owing to the insolubility of the molybdic acid complex of strychnine, no large variations of strychnine could be made and therefore the solution used was saturated with respect to strychnine.

The amount of hydrochloric acid necessary for the reagent, using commercial grades of molybdic acid or sodium molybdate, was very difficult to determine, for the reason that some grades of molybdic acid obtained contained as much as $1 \%$ of ammonia; a few samples of sodium molybdate were soluble in water, and others were not. Merck's sodium molybdate was found satisfactory, but difficult to obtain, so that the preparation of this substance by ourselves was necessary.

fo show the effect of varying amounts of strychnine and hydrochloric acid, the following experiments were made:

Strychnine.-To $1.5 \mathrm{~g}$. of sodilim molybdate, dissolved in 2.5 to $3.25 \mathrm{cc}$. of water, and to ce of hydrochloric acid ( 1,1$) \mathrm{sp}, \mathrm{gr} . \mathrm{I} .098$, were added $\mathrm{I} .0,0.75,0.50,0.25$ and o.oo ce. of a saturated solution of strychnine sulfate $(2 \%)$, respectively.

After shaking and standing over night, molybdic acid-strychnine crystallized out in all solutions, except the last. The one to which $0.25 \mathrm{cc}$. of strychnine sulfate had been added contained the least (only a small amonnt), while the others contained precipitates in proportion. The reagents were, after filtering, tested for their precipitating power nephelometrically, and it was found that the one without strychnine gave no test for phosphorus and that the others had about the same efficiency. A slight difference was observed, but before the question can be definitely settled the

: The suspensions always gave negative constants isee discussion of nephelometric constants is this paper).

Further work with this grade of sodium molybdate was impossible, since we were unable to fatain it cwing to the wur. 
experiments will have to be repeated. As our results below show, the reagent with I cc. of strychnine sulfate gave very satisfactory results, and until further data are at hand, it is best to adopt that amount.

Hydrochloric Acid.-To I $5 \mathrm{cc}$. of water, $2.5 \mathrm{cc}$. of reagent (see directions, given below) and $5.00 \mathrm{cc}$. of $\mathrm{KH}_{2} \mathrm{PO}_{4}$ solution $(5 \mathrm{mg}$. to a liter) were added varying amounts of normal hydrochloric acid and the volume made up to $40 \mathrm{cc}$. The table gives the nephelometric readings.

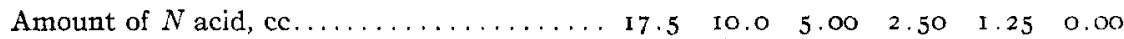

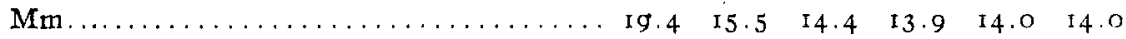

Since the amount adopted for regular work is $1.25 \mathrm{cc}$. $N$ under these conditions, almost four times as much may be used without decrease in the amount of precipitate. As may be seen, the results are also good when no acid is added in addition to that contained in the original reagent, thus showing a considerable latitude in the amount of free acid.

(b) Directions.-Preparation of Sodium Molybdate. 'Thirty-five and one-half grams of molybdic acid (c. P. free from ammonia, "IOO\%") are boiled for about one hour with $50 \mathrm{cc}$. sodium hydroxide solution (containing $400 \mathrm{~g}$. of $96 \% \mathrm{NaOH}$ dissolved in I liter). The volume of liquid is brought up to $84 \mathrm{cc}$. with distilled water and the mixture shaken, until practically all of the solid sodium molybdate is dissolved. After adding a few grams of pure talcum powder and again shaking, the solution is filtered free from any residue, which in some preparations may be very dark. The filtrate, which should not have more than a slight yellowish tint, is evaporated ${ }^{1}$ practically to dryness on the steam bath, and the residue washed, first, by grinding it with $40 \mathrm{cc}$. of $95 \%$ ethyl alcohol, filtering, and then by washing it on the filter with several portions of $20 \mathrm{cc}$. of alcohol. The residue, which should be perfectly white, is then dried in an oven at about $50^{\circ}$ or in a vacuum desiccator.

Strychnine Solution.-Two grams of pure strychnine sulfate are put into a Ioo cc. flask, with $80 \mathrm{cc}$. of water, and heated to about $90^{\circ}$. When the salt is completely dissolved, the solution is allowed to cool and then made up to the mark with distilled water.

Hydrochloric Acid ( $\mathrm{I}-\mathrm{I}$ ).-Fifty cubic centimeters of strong $\mathrm{HCl}$ (sp. gr. 1.20) are diluted to $100 \mathrm{cc}$. with water; $5.00 \mathrm{cc}$. of this acid diluted to roo cc. can be standardized by titrating with $0.5 N$ sodium carbonate. Twenty-four cc. of acid should be equivalent to $30 \mathrm{cc}$. of $0.5 \mathrm{~N}$ alkali.

Preparation of Reagent.-One and one-half grams of sodium molybdate (as prepared above) are dissolved in $2.5 \mathrm{cc}$. of distilled water and Io cc. of (I-I) hydrochloric acid added while shaking. The precipitate which is formed on the first additions of acid redissolves in the excess of acid. One cubic centimeter of strychnine sulfate solution is then added while shaking, and the solution allowed to stand over night. After filtering, the solution should be perfectly clear and practically colorless. For this

${ }^{1}$ After concentrating for sometime the solution forms a crust of sodium molybdate, which must be broken up occasionally with a stirring rod. 
purpose ordinary filter paper cannot be used, as the strongly acid solution extracts from it a substance which gives a very marked cloud on standing. This substance seems to be a phosphorus compound, as S \& S No. 575 , a hardened paper, and $\mathrm{S} \& \mathrm{~S}$ No. 589 a quantitative paper, do not seem to give the slightest trace even on standing for weeks. S \& S No. 597 gives a large anount of this suspension.

For Makn Standirt Solution.- One-tenth of a gram of $\mathrm{KH}_{2} \mathrm{PO}_{4}$ is dissolved in = liters of water; roo ce. of this stock solution diluted to I liter will make a sindard solution containing $5 \mathrm{mg}$. to a liter.

For precipitating. To $30 \mathrm{cc}$ of distilled water and $5 \mathrm{cc}$ of $0.5 \mathrm{~N} \mathrm{HCl}$, $5.00 \%$ or reagent are added and the solution shaken thoroughly. Ten c. of phosplate solution (standard or unknown) are ther added slowly with a pipet; the mixture is shaken by gentle rotation of the flask, and after 3 minutes' standing it is ready for reading in the nephelometer.

For frating the Instrument.- As these directions have already been given in detail, there is no need for repeating them here.

Results with New Reagent.--As Pouget and Chouchak studied the effect of different salts on their nitric acid reagent, the experiments with the new teagent were limited to pure solutions of mono-potassium phosphate, in series of liquids, containing $5.0,4.5,4.0,3.5,3.0$ and 2.5 mg. of phosphate, respectively, precipitates were produced as described in the directions and the readings plotted, as shown below.

Curve 1 Mig. 1) was made September II, 1914, with a reagent containing Merci's sodium molybdate and Curve B (Fig. 2) was made May 20, 1915 , with a reagent containing a preparation of our own sodium molybatate, ans indicated on page 2375 .

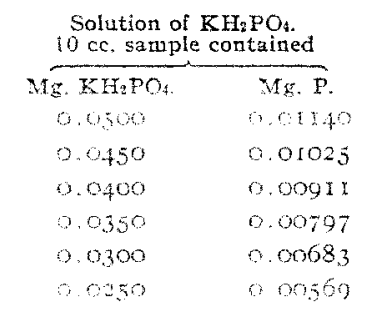

TABLE I.

\begin{tabular}{cccc}
\multicolumn{2}{c}{ Curve A. } & \multicolumn{2}{c}{ Curve B. } \\
$\begin{array}{c}\text { Readings. } \\
\text { Min. } \\
13.90\end{array}$ & $k$. & $\begin{array}{c}\text { Mrn. } \\
14.74\end{array}$ & $k$. \\
1.95 & 0.29 & 15.92 & 0.24 \\
15.40 & 0.23 & 17.26 & 0.23 \\
18.10 & 0.21 & 19.00 & 0.22 \\
19.90 & 0.21 & 20.90 & 0.22 \\
12.00 & 0.21 & 23.00 & 0.22 \\
& - & & \\
Average, & 0.23 & & 0.23
\end{tabular}

The figures represent the average of 2 to 3 readings upon two or three solutions of each concentration and the relatively large constant indicates that the reaction is practically complete. (See Discussion.)

\section{Discussion.}

In nephelometric work published from the Harriman Laboratory thus far, considerable stress has been laid on the position of the curves obtained

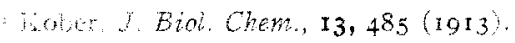


from a gradationed series of known solutions. These suspensions represent such small amounts of material that no other technic can be conveniently employed to determine the completeness of precipitation, and so far we have relied only on the position of these curves, relative to the colorimetric curves, to tell us whether the precipitation is complete or not. As these curves are 270 very important for rapid and ac-260 curate work, a recapitulation of 25.0 their meaning and use will not be out of place here.

Taking Fig. I as an example, 230 when the standard solution, after 22.0 precipitation, was put on both 21.0 sides of the instrument, the right side was set at $15.0 \mathrm{~mm}$. and when the light in the eye-piece was matched, the left side read I 3.92 (this reading, $\mathrm{I} 3.92$, is de- 17.0 noted by " $s$ " in the nephelome- 16.0 tric formula and curve). Now, leaving the standard solution ${ }^{1}$ on the right set at $55.0 \mathrm{~mm}$., another solution (0.900 as strong as the 13.0 (50 standard) was put in place of the standard on the left side, which

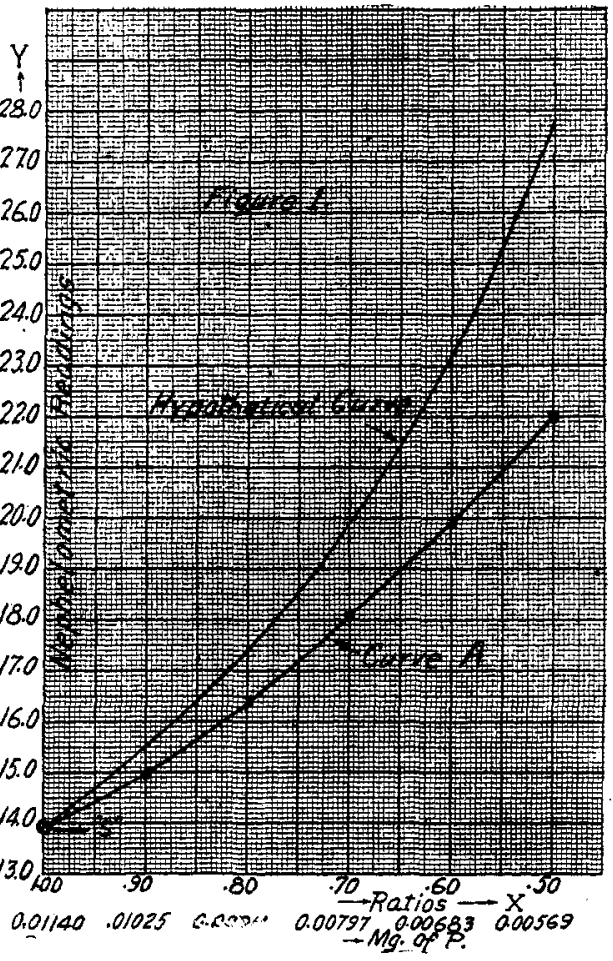
on being matched with the standard remaining on the right side, gave a reading of 14.95 (this and similar readings are denoted by " $y$ " in the formula and curve). Then solutions of $0.800,0.700,0.600$, and 0.500 standard strength were put on the left side and matched successively, thus giving, respectively, 16.40, 18.10, 19.90, and $22.00 \mathrm{~mm}$. A line drawn through these points gave us Curve $A$, and similarly Curve $B$ was obtained.

If the instrument and light remained constant so that the readings of the standard solution always remained the same, in this case, 13.92 mm., the curve would be sufficient for practical work. The readings of unknown solutions, by reference to the curve, could be expressed directly in milligrams of substance. But most instruments do not remain constant (not even a good balance) and therefore the readings of standard

${ }^{1}$ By using " $s$ " as the potential height of the standard liquid, any inequality in light, tubes, and plungers of the two sides is eliminated. It is similar to weighing by substitution. 
on the left side (the readings of the right side being the same, usually 15.0 mm.) changes, from time to time, similarly to the zero point of the balance, and the valne of the curve itself is thereby destroyed. Therefore,

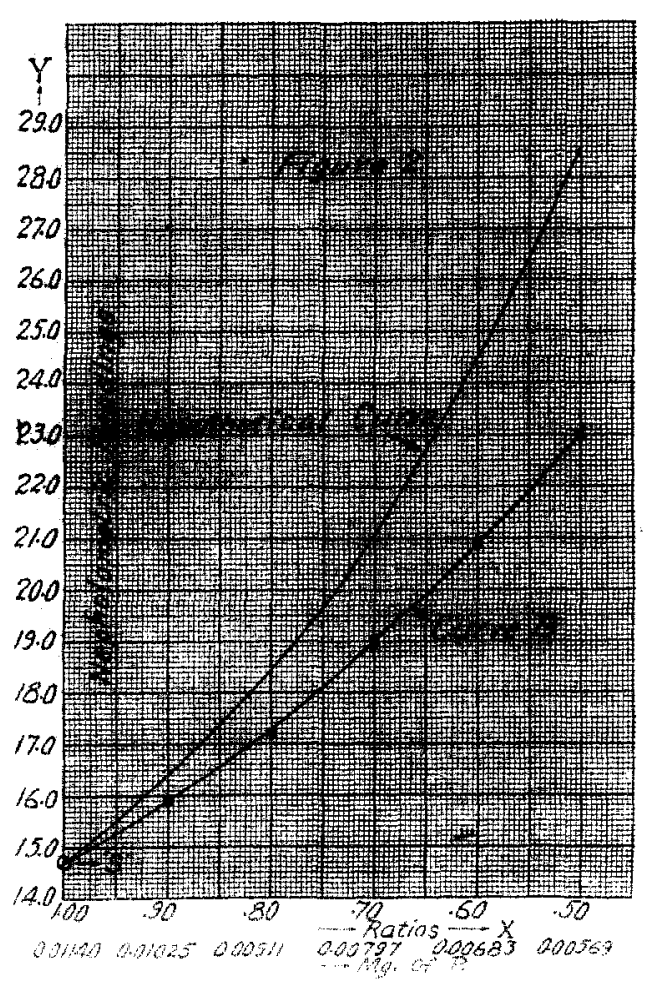

we always check up the "zeropoint" of the instrument, before beginning a series of analyses, by putting standard solution on iroth sides of the instrument and making readings.

To circumvent this difficulty of changing instrument, without using a nephelometric formula, two methods are available: (i) to redilute either the standard or the unknown solutions until they give the same readings. Unless the two readings are identical, which is difficult to obtain, an assumption or hypothesis must be introduced to finish the calculation, i. $e$, that the readings are inversely proportional to the concentration of the substance, as in the curve marked "hypothetical." How' croneous this assumption is and how near the rewdings of the "unknown" must be to those of the standard solutions to avold appreciable crror, can be seen at a glance by the difference

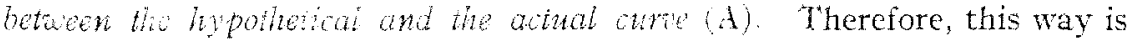
not only tedious, but not very accurate. (2) The second way to obviate the inconstancy of the instrument, is, of course, to make a fresh curve each time, which again is unnecessarily tedious.

If a nephelometric curve could be expressed by an equation, any change in any one of the variables (i. $e$., the readings) would be of no consequence, and in general, the beharior of the reagents could then be followed and expressed with ease. This has been attempted' in the formula

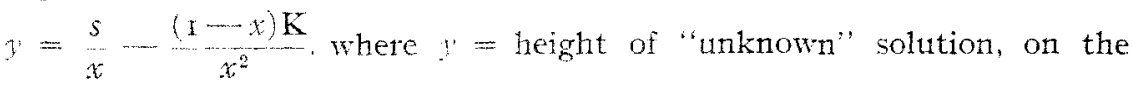
left side of the instrument, when standard solution is kept on the right side at a definite height, $s=$ height of standard solution on the left side and $:=$ the ratio of the concentrations of the two solutions. $K=a$

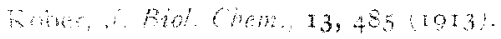


constant, obtained by substitution of standardization values of $s, y$, and $x$.

When $\mathrm{K}$ obtained with one height of standard solution is compared with that of another height, it is found that $\mathrm{K}$ is proportional to the height of the standard solution, and the equation then becomes for any heights of liquid within moderate limits

$$
y=\frac{s}{x}-\frac{(\mathrm{I}-x) s k}{x^{2}} \text { where } k=\frac{\mathrm{K}}{s} .
$$

When solved for $x$ the equation becomes

$$
x=\frac{s+s k+\sqrt{(s+s k)^{2}-4 s k y}}{2 y} .
$$

This equation, then, actually expresses the mathematical relationships between the ratios of the concentrations of the substances and the readings, and $k$, which is constant for a given substance and its reagent or precipitant, is an index of the amount of deviation of the readings from the colorimetric or hypothetical curve. Its value in the colorimetric curve is zero and the maximum thus far obtained for suspensions is 0.29 .

One of us (K.) conjunctively with Sara S. Graves has found it convenient to estimate the maximum deviation or constant possible from a given suspension. This can be obtained by precipitating a standard solution, then diluting an aliquot portion with more reagent to a definite volume, and comparing the two suspensions in the nephelometer. The readings of " $y$ " in this case will be slightly lower, and the constant slightly greater than the one used in actual analysis, or that obtained by diluting the solution before adding the reagent. This is due to the fact that when substances are precipitated in very dilute solutions, a small and often an appreciable amount remains unprecipitated, through its solubility or hydrolysis, but by precipitating before diluting, this solubility of the substance is eliminated, as both standard and "unknown" or weaker solution then contain, as under ideal conditions, the same proportion of precipitated and dissolved substance. ${ }^{1}$ We shall hereafter designate it as the theoretical constant of a substance, and the difference between it and the actual constant may then be taken as representing the amount of substance not precipitated, under the conditions of analysis.

With the Graves reagent for ammonia it was found that the theoretical constant was $0.26^{2}$ and the actual $0.144^{3}$ For this new reagent for phos-

${ }^{1}$ It is, of course, probable that the suspension in the weaker solution will redissolve or hydrolyse slightly, until the same equilibrium is reached as in the one precipitated after dilution, but the speed of solution, near the saturation point, as is well known, is extremely slow, and any error due to this must be negligible.

${ }^{2}$ From the note book of S. S. Graves, dated February 10, I9I 5.

${ }^{3}$ Even if the amount not precipitated is appreciable, no error in analysis results, as it is eliminated through standardization. 
phorus, the theoretical constant was found to be 0.23 , and the actual as shown above is near 0.22 (the average, including two high values, was 0.23 , which demonstrates that the precipitation of phosphorus in this extreme dihition is remarkably complete. The half standard solution contained i part of phosphorts in 2,000,000, while the final suspension in the nephelometer only contained one part in 10,000,000.)

Another point to be considered in connection with the completeness of precipitation is that of stability of the suspension. Experience has shown that the more complete a precipitation the less stable the suspotsion, i. . the quicker it agglutinates. Thus it is sometimes necessary to sacrifice somewhat on the completeness of the precipitation to gain stability of suspension (i. e, time for making readings). These and other properties of opalescent solutions will be taken up in detail in the near future

\section{Applications.}

our first intention was to apply the reagent to the estimation of phosphorus in various organic and inorganic substances, but lack of opportunity compels us to content ourselves with a few preliminary Hixs. As Pouget and Chouchak have already suggested and tried the nitric acid reagent for metals, ores and biological material, and Greenwald has shown that the same is useful for blood work, it will not be necessary to give many details and data for the purpose of showing its usefulness. The accuracy obtainable with the new reagent is shown by the rephelonetric ouves.

As the dilution of the solution for most phosphorus estimations is so large, wry tew substanes are likely to interfere with the reaction. In an extreme ase re-solution of a phosphomolybdate might be necessary. A: a rule, any solution containing phosphates, but no organic matter, may He used at once, provided the sohtion is neutral or shightly acid and free form antidity.

Wti o arine were digested, as in a Kjeldahl nitrogen estimation, wh $20 \mathrm{~m}$ of concentrated sulfuric acid and $10 \mathrm{~g}$. of potassium sulfate, min the solution was clear. After the melt had cooled it was dissolved th watw and made un to $500 \mathrm{ce}$. Twenty five ce. of the solution, neutralized roughly with ammonia and filtered, made up to $200 \mathrm{cc}$. gave a solution of suitable dilution for nephelonetric work. As this gave a suspension shghtly stronger than the standard used, it was put on the right side of the instrument and the standard on the left was used as an "unknown." The ratio of the diluted urine to the standard is therefore equal to the reciproce 1 or , in this case.

Jitration with uranium acetate gave 0.053 and $0.054 \mathrm{~g}$. of phosphorus

Fo masing these estmations. I an endebted to Dr. Thomas Koclair of Fraser

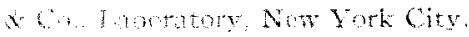


in I0O cc. of urine. Further work is of course nesessary to determine which of these values is the more accurate, and if the conditions for the application of the nephelometric method are satisfactory. It is possible that the ammonia used for neutralization has a slight inhibitory effect, and that sodium or lithium hydroxide will give better results.

TABLE II.

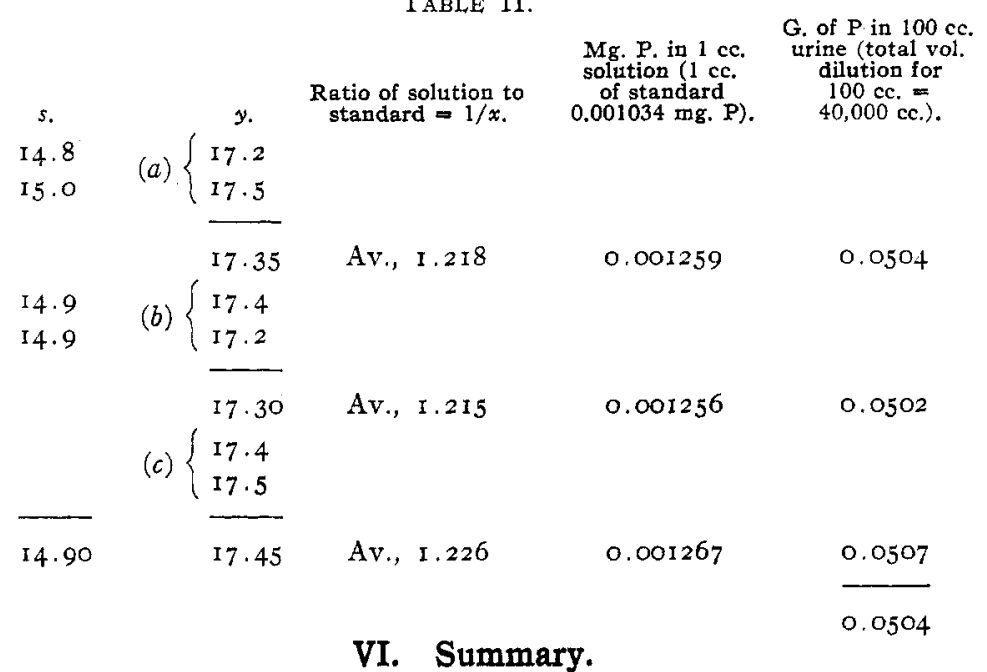

I. The reagent of Pouget and Chouchak has been modified, so as to be: (a) stable, (b) colorless, (c) quantitatively and (d) nephelometrically applicable.

2. It is shown that $0.005 \mathrm{mg}$. of phosphorus in Io cc. of solution, or one part of phosphorus in 2 million parts of water, is easily determined quantitatively with the nephelometer.

NEW YORK, N. Y.

NOTE.

Alternating Current Thermoregulators.-Davis ${ }^{1}$ has recently described an alternating current thermoregulator, designed for operation on the usual t Io v., 60 cycle lighting current. In my description of a large incubator ${ }^{2}$ a very similar system was described, differing mainly from Davis' in that a $200 \mathrm{ohm}$, main line, telegraph relay was used to break the circtit, this relay operating in series with a ro watt lamp and being controlled by a "fire-alarm" thermometer. This system operated very well for several months, the only trouble it gave being that incident to the sparking at the mercury-platinum contact of the thermometer; replacing the thermometer with a thermoregulator of larger bore improved

1 ThIS Journal, 37, 1520 (1915).

${ }^{2}$ J. Ind. Eng. Chem., 6, 939 (1914). 